\title{
Fratura de côndilos occipitais
}

\author{
Daniella Brito Rodrigues' ${ }^{1}$ Alzira Leite Gomes', Karlyne Palhares Brum', \\ Márcia Priscila de Oliveira Barbosa ${ }^{1}$, Bruno Tiago Barbosa Maia', \\ Eberval Gadelha Figueiredo' ${ }^{2}$, Manoel Jacobsen Teixeira ${ }^{3}$
}

Universidade do Estado do Pará (UEPA), Belém, PA, Brasil. Divisão de Clínica Neurocirúrgica do Hospital das Clínicas da Universidade de São Paulo (USP), São Paulo, SP, Brasil.

\section{RESUMO}

A fratura de côndilos occipitais é uma afecção considerada rara, mas que pode estar sendo subdiagnosticada. Fatores como a apresentação clínica variável, o exame físico frustro e a não identificação por radiografia simples dificultam esse diagnóstico, podendo levar a complicações como paralisia de nervos cranianos caudais e até mesmo a óbito. O presente estudo tem como objetivo revisar a literatura pertinente às fraturas de côndilos occipitais, com enfoque nas considerações anatômicas da junção craniocervical e ressaltando aspectos fisiopatológicos, parâmetros clínicos e as controvérsias quanto ao tratamento. $O$ incremento das técnicas radiológicas e a maior disponibilidade e uso de tomografia computadorizada possibilitaram o aumento do número de casos descritos dessas fraturas nas últimas décadas. A apresentação clínica é inespecífica e a tomografia da junção craniocervical é o método diagnóstico de escolha. A ausência de diagnóstico é responsável por sequelas, como déficits neurológicos, e foram descritas taxas de mortalidade de até $16 \%$ em casos de fraturas bilaterais. 0 mecanismo de injúria exato não é bem conhecido, mas a maioria dos autores indica a hiperextensão do pescoço associada à força vertical sobre a junção craniocervical. O tratamento é controverso, por causa da inconsistência nos resultados obtidos com o tratamento conservador baseado na classificação de Anderson e Montesano, em comparação com o escasso número de doentes tratados cirurgicamente.

\section{PALAVRAS-CHAVE}

Articulação atlantoccipital, osso occipital, cervicalgia, atlas cervical.

\section{ABSTRACT \\ Occipital condyles fractures}

The occipital condyle fractures are rare lesions, but they may have been under-diagnosed. Factors such as variable clinical presentation, inconclusive physical examination and no identification in the simple radiography difficult the diagnosis and may lead to complications such as paralysis of cranial nerves and death. This study aims to review the literature about occipital condyle fractures, emphasizing the anatomical considerations of the craniovertebral junction, pathophysiological view, clinical presentation and controversies regarding treatment. The improvement in radiological techniques and the increased availability and usage of computed tomography allowed the growth of reported cases of these fractures in recent decades. The clinical presentation is nonspecific and CT of the craniocervical junction is the diagnostic method of choice. The absence of a diagnosis is responsible for sequel, such as neurologic deficits, and as mortality rates are of up to $16 \%$ in cases of bilateral fractures. The exact mechanism of injury is not well known, but most authors indicate the hyperextension of the neck associated with the vertical force on the craniocervical junction. The treatment is controversial due to the inconsistencies in the results obtained with conservative treatment based on the classification of Anderson and Montesano, compared to the small number of patients treated surgically.

\section{KEYWORDS}

Atlanto-occipital joint, occipital bone, neck pain, cervical atlas.

1 Acadêmicos de Medicina da Universidade do Estado do Pará (UEPA), Belém, PA, Brasil.

2 Supervisor da Divisão de Clínica Neurocirúrgica e chefe do Grupo de Neurocirurgia Vascular do Hospital das Clínicas da Universidade de São Paulo (USP), São Paulo, SP, Brasil.

3 Diretor da Divisão de Clínica Neurocirúrgica do Hospital das Clínicas e professor titular da Disciplina de Neurocirurgia da USP, São Paulo, SP, Brasil. 


\section{Introdução}

A fratura de côndilo occipital (FCO) foi inicialmente descrita por Charles Bell, em 1817, durante a necrópsia de uma vítima de uma queda com traumatismo cranioencefálico grave. ${ }^{1}$ Já a primeira evidência radiográfica de uma fratura de côndilos occipitais in vivo foi relatada em $1962 .{ }^{2}$ Desde então, poucos casos têm sido descritos na literatura. ${ }^{3}$

A maior parte dos pacientes que sobreviveram à fratura de côndilos occipitais foi relatada na última década. Esse fato reflete a melhora do nível do atendimento de urgência, imobilização, transporte durante o atendimento inicial, novas técnicas de diagnóstico e maior índice de suspeição diagnóstica desse tipo de lesão. ${ }^{4}$

Fratura de côndilos occipitais deve ser sempre suspeitada em pacientes que sofreram trauma de alta energia envolvendo a cabeça ou a coluna cervical alta, resultando de carga axial, flexão e/ou rotação lateral e/ ou impacto direto nessa região. ${ }^{2}$ Essa fratura pode vir associada a lesões de nervos cranianos (31\%), sendo o hipoglosso o mais comumente envolvido (67\%). ${ }^{5}$

A apresentação clínica não é específica e a FCO não é prontamente diagnosticada durante o exame físico. ${ }^{2}$ Mesmo quando não associada a imediato déficit neurológico primário, pode evoluir para déficit neurológico de instalação tardia em decorrência da migração do fragmento ósseo fraturado ou de formação de calo ósseo. ${ }^{5}$

A falha no diagnóstico pode resultar em substancial morbidade, podendo gerar dor e limitação de movimentos, sérios déficits neurológicos ou até mesmo morte. É, portanto, mandatório um diagnóstico acurado, tanto para que se adote uma terapêutica adequada quanto por implicações médico-legais. Embora tenha sido tradicionalmente descrita como rara, mais recentemente, a fratura de côndilos occipitais tem sido considerada como uma condição subdiagnosticada e que ocorre com maior frequência do que é geralmente aceito. ${ }^{2}$

\section{Epidemiologia}

Essa afecção é mais prevalente em indivíduos do sexo masculino, tendo sido descrita em idades que variam desde os 6 aos 82 anos, no entanto a grande maioria dos doentes situa-se entre os 20 e 40 anos de idade. ${ }^{1,6}$

No passado, as FCO foram consideradas bastante raras por causa de sua difícil detecção com radiografia convencional. A primeira evidência radiográfica de uma FCO in vivo foi relatada em 1962, enquanto as primeiras tomografias computadorizadas (TC) foram publicadas em 1983. Desde então, com a ampliação do acesso à TC, os avanços tecnológicos dos exames de imagem e a melhoria no atendimento ao trauma, o número de notificações de FCO vem aumentando. O incremento das técnicas radiológicas e a maior disponibilidade e uso de TC possibilitaram o aumento do número de casos descritos de FCO nas últimas décadas. ${ }^{7-10}$ Capuano et al., ${ }^{11} \mathrm{em}$ uma revisão de literatura, encontraram somente 11 casos descritos entre 1817 e 1974, em detrimento dos 225 casos reportados a partir de 1999.

Estudos prospectivos com a realização de TC em doentes com traumatismo craniano grave (GCS entre 3 e 6) demonstraram incidência de $3 \%$ a $4,2 \%$ nessa população. ${ }^{1}$ Outro estudo, que ampliou os critérios de inclusão para incorporar todos os pacientes com mecanismos adequados de lesão, independente do grau na Escala de Coma de Glasgow, demonstrou incidência de $16 \%{ }^{2,7}$ Foram descritas incidências de $1 \%$ a 16\% em pacientes com traumas craniocervicais, no entanto sua verdadeira prevalência ainda permanece desconhecida. ${ }^{2,8,10} \mathrm{~A}$ maioria dos autores é unânime em assegurar uma subavaliação dessa patologia, tendo em conta a dificuldade do diagnóstico. ${ }^{1,2,9,11}$

\section{Anatomia da junção craniocervical}

A junção craniocervical (JCC) inclui o osso occipital $e$ as vértebras $\mathrm{C} 1 \mathrm{e} C 2$. Do ponto de vista anatômico, são consideradas as articulações entre occipital e atlas (articulação atlantoccipital) e entre atlas e áxis (atlantoaxial). Os principais movimentos na articulação $\mathrm{O}-\mathrm{C} 1$ são os de flexão-extensão, enquanto na C1-C2 é a rotação axial. Os ligamentos apical, alar, cruciforme, transverso do atlas, longitudinais anterior e posterior, atlantoaxial acessório, capsular, assim como as membranas atlantoccipitais anterior e posterior e a membrana tectória, são os responsáveis pela estabilidade dessa região. ${ }^{12}$

\section{Côndilos occipitais}

Os côndilos occipitais são duas proeminências laterais em relação ao forame magno, com as quais o crânio se articula com a coluna vertebral (Figura 1). A articulação atlantoccipital é formada pela junção da superfície convexa dos côndilos occipitais e as superfícies côncavas das facetas articulares superiores do atlas. A membrana tectória, o ligamento nucal e o ligamento cruciforme conferem estabilidade regional ao movimento de flexão da porção superior da coluna cervical. ${ }^{13,14}$ 


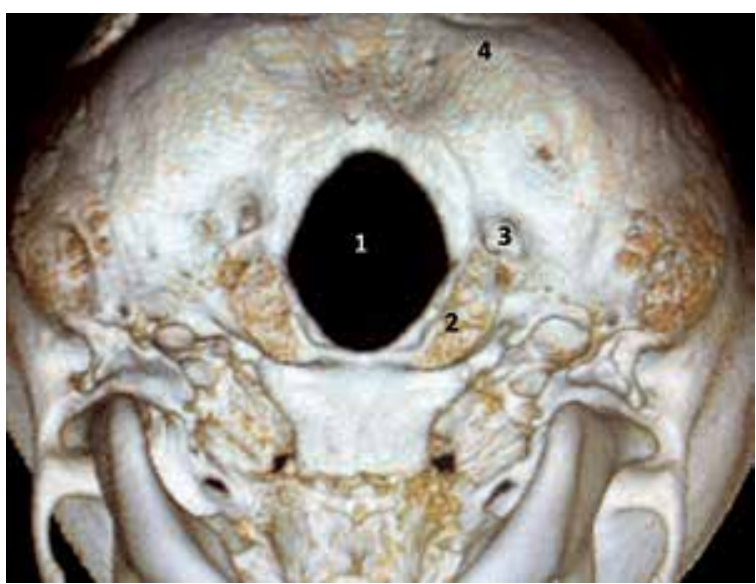

Figura 1 - Reconstrução 3D do crânio - (1) forame magno; (2) côndilo occipital; (3) fossa e canal condilar; (4) linha nucal inferior.

A importância clínica das fraturas de côndilos occipitais é justificada pela proximidade dos côndilos occipitais do bulbo, artérias vertebrais e nervos cranianos baixos. O bulbo, meninges, artérias vertebrais, artérias espinais anteriores e posteriores e as veias basivertebrais que se comunicam com o plexo venoso vertebral interno são estruturas que passam através do forame magno, o qual está intimamente relacionado com os côndilos occipitais. ${ }^{2}$

A artéria vertebral, após emergir da artéria subclávia e atravessar os forames transversos das seis primeiras vértebras cervicais, flete-se posterior e lateralmente em direção ao forame costotransverso do atlas, formando o segmento suboccipital, que contorna o arco posterior do atlas. O segmento suboccipital da artéria vertebral, após curto trajeto inclinado posterior à massa lateral do atlas, forma uma impressão sobre a superfície superior do arco do atlas, denominado sulco da artéria vertebral (SAV). O SAV marca com exatidão, nas vértebras isoladas, o local onde pulsa a artéria vertebral. ${ }^{15}$ As lesões do côndilo occipital estão frequentemente associadas à insuficiência da artéria vertebral. ${ }^{12}$

O canal do hipoglosso está intimamente relacionado com o côndilo occipital, pois essa pequena escavação está localizada na base do côndilo occipital; o nervo hipoglosso, o ramo meníngeo da artéria faríngea ascendente e uma veia emissária atravessam esse canal; enquanto o canal condilar, ao lado do forame magno, dá passagem às veias. Lateralmente ao côndilo occipital e posteriormente ao canal carotídeo, está localizado o forame jugular, dividido em pars nervosa e pars vascular, o qual abriga os nervos IX, X e XI, a veia jugular interna e a artéria meníngea posterior. ${ }^{2}$ É evidente, portanto, a estreita relação do côndilo occipital com essas nobres estruturas, o que explica o porquê de essas fraturas produzirem impactos sobre as estruturas vasculares, nervos cranianos e bulbo.

\section{Atlas}

C1, ou atlas, é uma vértebra única, porque não possui corpo vertebral nem processo espinhoso. É um anel ósseo que possui um par de massas laterais, responsáveis pela sustentação do peso do crânio em forma de globo. ${ }^{13}$ $\mathrm{O}$ atlas é a mais larga das vértebras cervicais, devido à origem de seus processos transversos, adjacentes às massas laterais, característica essa que proporciona maior alavanca aos músculos fixados. $\mathrm{Na}$ face superior do tubérculo do arco posterior, está localizado o sulco da artéria vertebral, o qual abriga também o nervo C1. As faces articulares superiores das massas laterais recebem duas grandes protuberâncias cranianas denominadas côndilos occipitais. ${ }^{13}$

Cerca de um terço do anel do atlas é ocupado pelo processo odontoide, um terço por um espaço intermediário e um terço pela medula espinhal - regra dos terços de Steele -, havendo, assim, suficiente espaço para a luxação posterior do odontoide. Isso explica a ausência de sintomas em alguns deslocamentos do atlas até que haja alto grau de movimento (maior que um terço do diâmetro do anel do atlas). ${ }^{13}$

\section{Áxis}

É a mais forte das vértebras cervicais; $\mathrm{C} 1$, que sustenta o crânio, gira sobre C2. Possui duas faces articulares superiores, sobre as quais o atlas gira; seu processo espinhoso é bífido e pode ser palpado profundamente no sulco nucal. A característica mais marcante do áxis é o processo odontoide, o qual se projeta do seu corpo para cima, está situado anteriormente à medula espinhal e serve como eixo em torno do qual ocorre a rotação. $\mathrm{O}$ processo odontoide é mantido contra a face posterior do arco anterior do atlas pelo ligamento transverso do atlas. Esse ligamento, por sua vez, estende-se de uma massa lateral à outra do atlas, servindo como anteparo entre o processo odontoide e a medula espinhal. Desse modo, impede o deslocamento posterior do processo odontoide e o deslocamento anterior do atlas. É importante ressaltar que o suprimento sanguíneo da porção superior do processo odontoide é fornecido pelo ramo da artéria do hipoglosso, enquanto sua base é irrigada por ramos da artéria vertebral..$^{12}$

\section{Articulações craniovertebrais}

O crânio e a coluna vertebral encontram-se unidos por uma série de articulações envolvendo o occipital, 
o atlas e o áxis. As articulações craniovertebrais são articulações sinoviais que não possuem discos intervertebrais. São articulações craniovertebrais: a articulação atlantoccipital e a atlantoaxial. ${ }^{1}$

A articulação atlantoccipital ocorre entre os côndilos occipitais e as cavidades glenoideias do atlas, situadas na face superior das massas laterais da primeira vértebra cervical. O crânio e $\mathrm{C} 1$ estão unidos por membranas atlantoccipital anterior, posterior e ligamentos atlantoccipitais laterais. A membrana tectória é a continuação superior do ligamento longitudinal posterior e descreve um trajeto de $\mathrm{C} 2$ até a base do crânio. ${ }^{12}$

A rotação axial e a translação anteroposterior entre $\mathrm{C} 0-\mathrm{C} 1$ são controladas pelos ligamentos apicais e ligamentos alares, enquanto o ligamento transverso limita a translação anteroposterior do complexo $\mathrm{C} 0-\mathrm{C} 1$ sobre $\mathrm{C} 2$. As artérias vertebrais penetram na membrana atlantoccipital posterior antes de atravessarem o forame magno. ${ }^{12}$

\section{Mecanismos de injúria}

Conforme a abordagem anatômica anteriormente descrita, é facilmente perceptível que a estabilidade da JCC depende muito mais da integridade das estruturas ligamentares que das estruturas restantes. Ademais, a instabilidade da JCC é resultante de lesões por destruição de uma série de ligamentos, em ambas as articulações: atlantoaxial e atlantoccipital. $\mathrm{Na}$ articulação atlantoccipital, as estruturas mais importantes para a estabilidade mecânica são a membrana tectória e os ligamentos alares emparelhados. ${ }^{2}$

Outra consideração importante é que o traumatismo envolvendo a JCC também é influenciado pela posição da massa e do crânio em relação ao eixo longitudinal da coluna vertebral cervical, no momento da lesão. Raramente, é uma carga axial direta fornecida à coluna em si, mas, sim, é transferido a partir da base do crânio para baixo através da coluna cervical. A localização da força aplicada ao crânio determina as forças transferidas para a coluna cervical (estes incluem carga axial ou carga axial assimétrica com as forças de flexão laterais simétricas ou assimétricas, aplicadas ao occipital posteriormente, e forças de hiperextensão ou hiperflexão, em associação com a distração e as forças de rotação lateral). No entanto, lesões associadas são extremamente frequentes. Por exemplo, em acidentes automobilísticos, principalmente colisões traseiras, a cabeça, ligeiramente rodada inicialmente, vai entrar em rotação máxima seguida por um movimento de "chicotada" causado pelo impacto. Em particular, nesse mecanismo de lesão, os ligamentos alares, os quais limitam a rotação axial, são os mais vulneráveis. ${ }^{2}$
Finalmente, a configuração da JCC é resultante dos diâmetros sagital e transversal normais do forame magno e do canal espinal cervical (a parte superior, a qual é mais larga do que a parte inferior com relativamente maior espaço para a medula espinhal superior), o que explica como lesões traumáticas com deslocamento de fragmento podem ocorrer com menos déficits neurológicos do que em lesões traumáticas da coluna cervical baixa. Graves lesões craniocervicais, com ou sem deslocamentos atlantoccipital substanciais, apesar de instáveis, podem ocorrer sem dano neurológico. No entanto, isso é complicado, pelo fato de que uma massiva lesão na cabeça e um trauma intracraniano muitas vezes acompanham lesões cervicais superiores. Dessa forma, o mecanismo exato da FCO não é bem conhecido, mas a maioria dos autores indica como provável a hiperextensão do pescoço associada à força vertical sobre a JCC., ${ }^{2,4}$

A secção dos ligamentos alares e da membrana tectória permite o deslocamento anterior do crânio com relação à coluna vertebral. É necessária a ruptura completa de todas as estruturas ligamentares entre o occipício e o complexo atlantoaxial para que ocorra a luxação traumática atlantoccipital (LTAO). Os ligamentos alares estão mais distendidos e, consequentemente, mais vulneráveis à lesão quando a cabeça está rodada e, adicionalmente, fletida. Quando há luxação, autores acreditam que a hiperextensão, causando a ruptura da membrana tectória, seja a causa principal, enquanto outros consideram que o componente de flexão lateral também seja necessário. ${ }^{4}$

O mecanismo da lesão neurovascular é atribuído à compressão ou ao estiramento dos nervos, lesão direta do tronco encefálico ou insuficiência da artéria vertebral. O mecanismo de tração axial, sofrida pela medula e nervos cranianos como consequência da separação entre os côndilos occipitais e o atlas, seria o responsável pela presença de lesões dos nervos cranianos, principalmente paralisia do IX, X, XI e XII pares, frequente nos pacientes com LTAO. Foram relatadas lesões secundárias das artérias vertebrais, incluindo compressão, lesão da íntima ou trombose. A lesão é instável e pode ocorrer piora do quadro neurológico quando o diagnóstico não é realizado na fase aguda. ${ }^{4}$

Foram descritas taxas de mortalidade de até $16 \%$ nesses tipos de fraturas, embora esse número reduza para $8,3 \%$ quando se analisam somente os casos de fraturas unilaterais sem evidência de LTAO. ${ }^{16}$

\section{Sistemas de classificação}

A classificação mais utilizada é a de Anderson e Montesano, ${ }^{17}$ de 1988 , que correlaciona o mecanismo 
do trauma com a morfologia da fratura. Foram descritos três tipos com base na morfologia e no mecanismo do trauma:

- Tipo I: fratura por compressão axial, impactação e fragmentação do côndilo, fratura cominutiva do côndilo; é considerada estável em razão da normalidade da membrana tectória e do ligamento alar contralateral, a menos que seja bilateral e/ou associada a deslocamento;

- Tipo II: fratura do côndilo linear contínua com fratura basioccipital; pode ser bilateral (Figura 2); o mecanismo de lesão é o trauma direto no crânio. A membrana tectória e ligamentos alares intactos preservam a estabilidade; se o côndilo estiver separado do crânio, pode ocorrer instabilidade e o tratamento com halocolete é recomendado por seis a oito semanas;

- Tipo III: fratura de avulsão do côndilo no ponto de inserção do ligamento alar; é potencialmente instável; o mecanismo de produção é a rotação forçada e a flexão lateral ou a associação dos dois movimentos; com a avulsão na região de inserção de um ligamento alar, a membrana tectória e o ligamento alar contralateral podem ficar sobrecarregados e romper, possibilitando deslocamentos. Nesse caso, devido à lesão do ligamento alar e da membrana tectória, observa-se uma lesão potencialmente instável. O tratamento depende da existência ou não de deslocamento atlantoaxial; na avulsão do ligamento alar bilateral, pode ocorrer deslocamento craniocervical em $30 \%$ a $50 \%$ dos casos. Este último tipo pode causar dor e limitação de movimentos e estar associado à lesão dos nervos cranianos baixos. ${ }^{18}$

Posteriormente, Tuli et al. ${ }^{19}$ propuseram, em 1997, uma nova classificação da FCO, que se baseia no grau de lesão ligamentar refletido pela presença de deslocamento da FCO e determinação da instabilidade das articulações O-C1-C2, observados no exame radiológico simples da coluna cervical, na TC e na ressonância magnética (RM)..$^{18}$

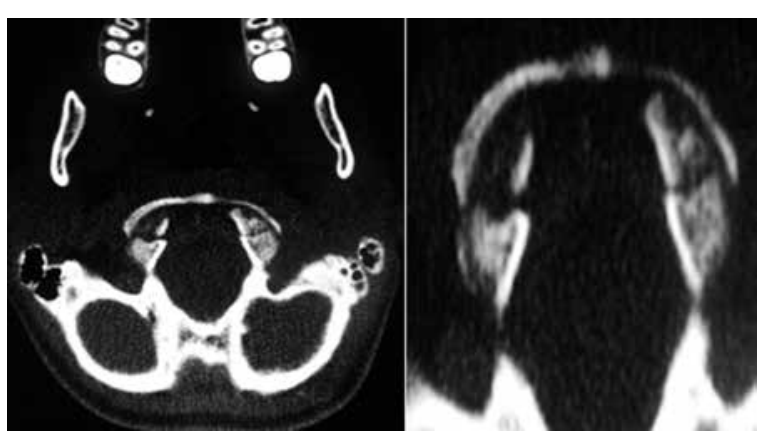

Figura 2 - Tomografia computadorizada de crânio - corte axial. Fratura bilateral dos côndilos occipitais, tipo II de Anderson e Montesano.
Essa nova classificação inclui os seguintes tipos de FCO:

- Sem deslocamento;

- $\quad 2 \mathrm{~A}$ - com deslocamento e estabilidade do complexo articular O-C1- C2;

- $2 \mathrm{~B}$ - com instabilidade do complexo articular $\mathrm{O}-\mathrm{C} 1-\mathrm{C} 2$.

A classificação de Tuli et al., ${ }^{19}$ baseada no grau de lesão ligamentar, é mais funcional e retira a ênfase da anatomia do côndilo occipital para colocá-la na mobilidade funcional do complexo articular O-C1-C2. As FCO tipos 1 e 2 de Anderson e Montesano ${ }^{17}$ correspondem ao tipo 1 de Tuli et al. ${ }^{19}$ A FCO tipo 3 de Anderson e Montesano corresponde ao tipo 2 de Tuli et al., que é subclassificada em A e B na dependência da estabilidade ou instabilidade do complexo articular O-C1-C2. ${ }^{20}$

Segundo Paiva et al., ${ }^{21}$ essa classificação pode guiar o manejo neurocirúrgico, de modo que a fratura tipo $2 \mathrm{~B}$ requer instrumentação cirúrgica ou halo de tração; a fratura tipo $2 \mathrm{~A}$ deve ser tratada com um colar rígido; e a fratura tipo 1 não requer tratamento específico.

Em 2012, Mueller et al. ${ }^{16}$ propuseram uma nova classificação para a fratura dos côndilos occipitais baseada no estudo prospectivo com seguimento de 31 doentes por um período de cinco anos:

- Tipo I - fratura unilateral sem luxação atlantoccipital;

- Tipo II - fratura bilateral dos côndilos sem luxação atlantoccipital;

- Tipo III - fratura unilateral ou bilateral dos côndilos com luxação atlantoccipital.

Para esses autores, somente o tipo III necessita de tratamento cirúrgico, considerando a grande maioria dessas fraturas inerentemente estável. ${ }^{16}$

A lesão dos nervos cranianos ocorre em cerca de $31 \%$ dos casos, sendo primária em $62 \%$ das ocasiões. Todas as formas secundárias (38\%) relatadas surgiram na sequência de tratamentos conservadores em lesões consideradas estáveis segundo Anderson e Montesano. ${ }^{17} \mathrm{~A}$ explicação para essa observação poderá residir no envolvimento dos nervos num processo cicatricial num contexto de instabilidade, tornando discutível a orientação terapêutica segundo os critérios de Anderson e Montesano.

\section{Apresentação clínica}

A apresentação clínica dos pacientes com uma FCO é altamente variável e vai desde cervicalgia com limitação de movimento até óbito por luxação atlantoccipital. ${ }^{20} \mathrm{E}$ os mais graves déficits neurológicos relatados em pacientes com FCO parecem estar relacionados com 
a severidade de traumatismos cranianos, e não com a própria $\mathrm{FCO}^{3}$

As complexas estruturas neurovasculares próximas à articulação O-C1 explicam a sintomatologia da luxação ou da FCO. Essa fratura pode estender-se até o canal do hipoglosso, localizado na base do côndilo occipital, ou até o forame jugular, localizado lateralmente ao côndilo, determinando paralisia de um ou mais dos quatro últimos nervos cranianos caudais. ${ }^{20}$ Portanto, a manifestação clínica também depende do nível e da gravidade da lesão. Pode variar desde a parada cardiorrespiratória com tetraplegia a exame neurológico normal; algumas vezes, o paciente apresenta somente desconforto e rigidez cervical. ${ }^{10}$

Embora a maioria dos pacientes com FCO isolada se apresente sem déficit neurológico, é importante levar em consideração que essa fratura pode estar associada a déficit neurológico de instalação imediata ou tardia. O mais frequente é a paralisia dos nervos cranianos caudais em variadas combinações, indo desde a paralisia isolada do hipoglosso até a paralisia de todos os nervos cranianos caudais, do IX ao XII par craniano - síndrome de Collet-Sicard. Nesses casos, a paralisia dos nervos pode apresentar-se tardiamente, talvez por mobilização de um fragmento de osso fraturado que não foi devidamente estabilizado inicialmente ou por formação de calo ósseo. ${ }^{19}$ Os sobreviventes com ausência completa de lesão neurológica são raros. ${ }^{3}$

Apesar da falta de especificidade do quadro clínico de FCO, a literatura indica alguns parâmetros que, quando presentes em vítimas de traumatismo cranioencefálico ou da coluna cervical, devem sugerir a hipótese dessa lesão e indicar a realização de TC da JCC. Esses parâmetros são: cervicalgia persistente mesmo com radiografia da coluna cervical normal, torcicolo, paralisia de um ou mais dos quatro últimos nervos cranianos caudais, edema pós-traumático retrofaríngeo ou prévertebral e fratura e/ou luxação da coluna cervical alta. ${ }^{3}$

\section{Exames de imagem}

A radiografia simples de crânio na incidência lateral ou anteroposterior não demonstra a FCO, em razão da superposição de estruturas adjacentes da região mastoideia e mandibular. ${ }^{18}$ Então, é importante saber de antemão que a visualização desse tipo de fratura com as técnicas radiográficas habituais é extremamente difícil, sendo comumente necessário o uso de tomografia, que é a técnica de escolha para seu diagnóstico. Cortes finos $(1,2 \times 1 \mathrm{~mm})$ permitem ótimo estudo de morfologia óssea e ligamentar, além de permitirem excelente reconstrução tridimensional. Logo, o diagnóstico definitivo é feito com TC convencional ou helicoidal, com cortes finos e reconstrução em plano sagital e coronal. ${ }^{22-26}$

A TC de alta resolução, com reconstruções sagital e coronal, constitui o método de escolha, porque permite tornar visível a base do crânio e não requer a movimentação do paciente. Entretanto, se não existe instabilidade craniocervical, o corte coronal direto com janela óssea permite a demonstração precisa da FCO. ${ }^{20,24,27}$ Além disso, a RM de coluna cervical pode complementar o estudo, por avaliar a integridade ligamentar e a compressão ou lesão medular. A RM complementa a investigação, demonstrando a relação entre o fragmento ósseo, o tronco encefálico e as cisternas da fossa posterior. Pode também evidenciar a ruptura de ligamentos, possibilitando a avaliação da estabilidade da JCC. ${ }^{27,28}$

\section{Tratamento}

Não há consenso na literatura sobre o tratamento das FCO. A controvérsia ocorre em virtude da inconsistência nos resultados obtidos com o tratamento conservador baseado na classificação de Anderson e Montesano, em comparação com o escasso número de doentes tratados cirurgicamente. ${ }^{1} \mathrm{O}$ tratamento conservador dessas fraturas evolui com bons resultados, com melhora da cervicalgia e manutenção dos movimentos do segmento envolvido após três meses de tratamento. ${ }^{5,21,29}$

A maioria dos casos publicados foram tratados conservadoramente, principalmente aqueles com fraturas classificadas em tipos I e II de Anderson e Montesano. ${ }^{17}$ Entretanto, a análise dos resultados do tratamento conservador de acordo com essa classificação tem sido controversa. A morbidade em termos de dor e aparecimento de comprometimento neurológico tardio tem levado alguns autores a questionarem os critérios de estabilidade descritos na classificação de Anderson e Montesano, apontando-a como excessivamente morfológica, não traduzindo o real grau de instabilidade desse tipo de lesão. ${ }^{16}$

$\mathrm{Na}$ revisão da literatura produzida nas diretrizes da American Association of Neurological Surgeons, identificou-se que 23 pacientes não receberam tratamento (2 com fraturas do tipo I; 14 do tipo II; 5 do tipo III e 2 com fraturas não classificadas), enquanto 44 pacientes foram tratados com imobilização com colar cervical. Treze pacientes receberam halo-minerva, e em seis a imobilização não foi descrita. Nessa revisão, apenas cinco pacientes foram operados. Dois pacientes com fratura tipo III foram submetidos à fixação occipitocervical (um com deslocamento atlantoccipital e um com instabilidade atlantoaxial), e três pacientes foram submetidos 
à descompressão de fragmento, com melhora imediata dos sintomas em um deles. ${ }^{18}$ Portanto, a problemática da opção terapêutica ideal é uma questão ainda a ser esclarecida. O estudo recentemente publicado por Mueller et al. ${ }^{16}$ propõe uma nova classificação, mais simplificada, em que o tratamento cirúrgico apenas tem lugar no caso de fratura dos côndilos associada à luxação atlantoccipital. Esses autores advogam tratamento conservador com colar cervical para a grande maioria desse tipo de lesões. Para Mueller et al. ${ }^{16}$ a aplicação do halo-vest não é necessária em pacientes com FCO unilateral sem luxação atlantoccipital, como tem sido postulado em outros estudos. ${ }^{30}$

Para Gusmão et al. ${ }^{20}$ além do critério de correção da instabilidade da articulação O-C1, deve-se levar também em consideração, para a indicação cirúrgica, a compressão do sistema nervoso, como nos casos de instabilidade $\mathrm{O}-\mathrm{C} 1$ por fratura associada ao deslocamento do côndilo fraturado para o interior do forame magno, no qual há significativos déficits neurológicos. Outros autores também indicam intervenção cirúrgica nas fraturas instáveis acompanhadas de complicações agudas do tipo compressão neurovascular. ${ }^{1,5}$ Já outros estudos preconizam tratamento conservador (halocolete, tração cervical e colar cervical) até mesmo nas fraturas instáveis acompanhadas por lesões nervosas, observando grande porcentagem de recuperação funcional. ${ }^{16}$ Segundo Botelho et al. ${ }^{18}$ tratamentos adicionais à imobilização devem ser individualizados de acordo com a presença de fraturas cervicais associadas, instabilidade e compressões neurais.

O tratamento cirúrgico da FCO isolada (não associada a deslocamento $\mathrm{O}-\mathrm{C} 1$ ) foi indicado com o objetivo de descompressão e estabilização. $\mathrm{O}$ deslocamento do côndilo occipital, em muitos casos, resulta em sintomas como hemiparesia, síndrome de Claude Bernard-Horner, síndrome de Collet-Sicard, entre outros. ${ }^{20} \mathrm{Se}$ a condição clínica do paciente politraumatizado permitir, a fixação occipitocervical é altamente necessária nos casos de luxação traumática atlantoccipital, por causa da alta instabilidade. ${ }^{31}$ Foram descritas taxas de mortalidade de até $16 \%$ em pacientes com FCO bilaterais, em oposição à prevalência de $8,3 \%$ em pacientes com $\mathrm{FCO}$ unilateral, sem evidências de luxação atlantoccipital traumática. ${ }^{16}$

\section{Considerações finais}

A fratura do côndilo occipital é relativamente pouco frequente e de difícil diagnóstico. Encontra-se bem descrita do ponto de vista do quadro clínico e dos exames complementares, sendo ainda controverso o seu tratamento, especialmente no que se refere à indicação e ao tipo de tratamento cirúrgico. A determinação da real incidência de FCO é difícil, porque o paciente politraumatizado pode ser assintomático e porque essa condição pode ser mascarada pela morte ou por lesões concomitantes, ou ainda por poder apresentar manifestações retardadas. No entanto, a FCO não deve ser considerada rara, uma vez que pode ocorrer em até $16 \%$ de pacientes com lesão craniocervical. O grande potencial de morbidade em longo prazo, relacionado à dor, à limitação de movimentos, a déficits neurológicos graves ou até mesmo à morte, justifica a crescente terapêutica e as implicações médico-legais de um acurado diagnóstico dessa afecção. $\mathrm{O}$ tratamento adequado na fase aguda permite a realização do tratamento definitivo, que pode conduzir à reabilitação do paciente e ao retorno às suas atividades normais após a ocorrência de uma lesão de alta gravidade e que poderia evoluir para óbito ou para uma lesão neurológica irreversível.

\section{Referências}

1. Brito JS. Fraturas de côndilos occipitais. Rev Port Ortop Traum. 2013;21(1):63-70.

2. Leone A, Cerase A, Colosimo C, Lauro L, Puca A, Marano P. Occipital condylar fractures: a review. Radiology. 2000;216(3):635-44.

3. Noronha HG, Letaif OB, Rocha ID, lutaka AS, Cristante AF, Oliveira RP, et al. Fraturas do côndilo occipital: atualização da experiência em nosso serviço e revisão da literatura. Coluna/Columna. 2013;12(2):157-9.

4. Defino H, Porto MA, Herrero CFPS, Romeiro CFWE, Barbosa MHN. Luxação traumática atlantoccipital: relato de caso. Rev Bras Ortop. 2008;43(5):203-8.

5. Falavigna A, da Silva FM, Hennemann AS. Occipital condyle fracture associated with Jefferson's fracture and injury of lower cranial nerves: case report. Arq Neuropsiquiatr. 2002;60(4):1038-41.

6. Strehle EM, Tolinov V. Occipital condylar fractures in children: rare or underdiagnosed? Dentomaxillofac Radiol. 2012;41(2):175-6.

7. Alcelik I, Manik KS, Sian PS, Khoshneviszadeh SE. Occipital condylar fractures. Review of the literature and case report. J Bone Joint Surg Br. 2006;88(5):665-9.

8. Dashti R, Ulu MO, Albayram S, Aydin S, Ulusoy L, Hanci M. Concomitant fracture of bilateral occipital condyle and inferior clivus: what is the mechanism of injury? Eur Spine J. 2007;16(Suppl 3):261-4.

9. Hadley MN, Walters BC, Grabb PA, Oyesiku NM, Przybylski GJ, Resnick DK, et al. Occipital condyle fractures. Neurosurgery. 2002;50:114-9.

10. Ide C, Nisolle JF, Misson N, Trigaux JP, Gustin T, De Coene $B$, et al. Unusual occipitoatlantal fracture dissociation with no neurological impairment. Case report. J Neurosurg. 1998;88(4):773-6.

11. Capuano C, Costagliola C, Shamsaldin M, Maleci A, Di Lorenzo N. Occipital condyle fractures: a hidden nosologic entity. An experience with 10 cases. Acta Neurochir (Wien). 2004;146(8):779-84. 
12. Baaj AA, Mummaneni PV, Uribe JS, Vaccaro AR, Greenberg MS, editors. Handbook of spine surgery. New York: Stuttgart; 2012.

13. Moore KL, Dalley AFA, Anne MR. Anatomia orientada para a clínica. $6^{a}$ ed. Rio de Janeiro: Guanabara Koogan; 2011.

14. Dvorak J, Panjabi MM. Functional anatomy of the alar ligaments. Spine (Phila Pa 1976). 1987;12(2):183-9.

15. Hasan M, Shukla S, Siddiqui MS, Singh D. Posterolateral tunnels and ponticuli in human atlas vertebrae. J Anat. 2001;199(Pt 3):339-43.

16. Mueller FJ, Fuechtmeier B, Kinner B, Rosskopf M, Neumann $\mathrm{C}$, Nerlich M, et al. Occipital condyle fractures. Prospective follow-up of 31 cases within 5 years at a level 1 trauma centre. Eur Spine J. 2012;21(2):289-94.

17. Anderson PA, Montesano PX. Morphology and treatment of occipital condyle fractures. Spine (Phila Pa 1976). 1988;13(7):731-6.

18. Botelho RV, Borgheresi MD, Batista AL. Trauma raquimedular craniocervical. Arq Bras Neurocir 2008;27(4):122-35.

19. Tuli S, Tator CH, Fehlings MG, Mackay M. Occipital condyle fractures. Neurosurgery. 1997;41(2):368-76.

20. Gusmão SS, Silveira RL, Arantes A. Surgical treatment of the occipital condyle fracture: case report. Arq Neuropsiquiatr. 2001;59(1):134-7.

21. Paiva WS, Rusafa-Neto E, Amorim RL, Figueiredo EG, de Andrade AF, Teixeira MJ. Occipital condyle fracture in a patient with head trauma. Arq Neuropsiquiatr. 2009;67(1):119-20.

22. Bloom Al, Neeman Z, Slasky BS, Floman Y, Milgrom M, Rivkind $A$, et al. Fracture of the occipital condyles and associated craniocervical ligament injury: incidence, CT imaging and implications. Clin Radiol. 1997;52(3):198-202.

23. Clayman DA, Sykes CH, Vines FS. Occipital condyle fractures: clinical presentation and radiologic detection. AJNR Am J Neuroradiol. 1994;15(7):1309-15.
24. Nogueira-Barbosa MH, Defino HL. Multiplanar reconstructions of helical computed tomography in planning of atlanto-axial transarticular fixation. Eur Spine J. 2005;14(5):493-500.

25. Weissman JL. Condylar canal vein: unfamiliar normal structure as seen at CT and MR imaging. Radiology. 1994;190(1):81-4.

26. Deliganis AV, Baxter AB, Hanson JA, Fisher DJ, Cohen WA, Wilson AJ, et al. Radiologic spectrum of craniocervical distraction injuries. Radiographics. 2000;20:237-50.

27. Young WF, Rosenwasser RH, Getch C, Jallo J. Diagnosis and management of occipital condyle fractures. Neurosurgery. 1994;34(2):257-60.

28. Urculo E, Arrazola M, Arrazola M Jr, Riu I, Moyua A. Delayed glossopharyngeal and vagus nerve paralysis following occipital condyle fracture. Case report. J Neurosurg. 1996;84(3):522-5.

29. Schrödel MH, Kestlmeier R, Trappe AE. Bilateral occipital condyle fracture: report of two cases. Skull Base. 2002;12(2):93-6.

30. Malham GM, Ackland HM, Jones R, Williamson OD, Varma DK. Occipital condyle fractures: incidence and clinical follow-up at a level 1 trauma centre. Emerg Radiol. 2009;16(4):291-7.

31. Maserati MB, Stephens B, Zohny Z, Lee JY, Kanter AS, Spiro RM, et al. Occipital condyle fractures: clinical decision rule and surgical management. J Neurosurg Spine. 2009;11(4):388-95.

\section{Endereco para correspondência}

Daniella Brito Rodrigues

Rua Silva Castro, 559, Guamá

66075-104 - Belém, PA, Brasil

Telefone: (91) 8815-7806

E-mail: britodaniella32@gmail.com 